\section{Software buy-out}

Goodteith Software has been acquired by Dentally, the UK's leading cloud dental software, marking the beginning of a new chapter for Goodteith and their customers.

Goodteith has been supplying Scottish dental practices with clinical software since 2000, and was designed and developed by Alistair Johnston, principal dentist at the Callander Dental Practice. It is used by more than 50 dental practices across Scotland.

Nick Davies, Co-Founder and Chief Executive Officer of Dentally, said: 'We are delighted to have welcomed Goodteith to our business, which we felt was a great match to our ethos. We have common values and Alistair shares our passion for providing dentists with an easy to use patient management tool that allows them to offer the highest level of care to patients.'

Alastair Johnston, Founder of Goodteith, said: 'We have had 18 successful years with Goodteith, but after much consideration felt that our platform was not the best choice for dental practitioners in Scotland to embrace the changes that NHS Scotland is undergoing. We invested time in looking at another system for our customers and felt Dentally was the best choice.

'I ran my own practice for 35 years and over the years have had the opportunity to work closely with 70 practices who have been Goodteith customers. Dentally has been around for a few years and have clearly listened to the dentists they work with in designing their software.

'They have a technically solid and friendly team of 12 that genuinely want to help dentists get on with their jobs. The software is easy-to-use, well thought out, practical and far more future-proof than other platforms we looked at.'

Dentally's cloud dental software has been designed in collaboration with dentists across the UK to create a hassle-free experience that allows dentists to work from anywhere, streamline their practice and free up valuable time.

Over the next 12 months, Dentally will continue to run and support the Goodteith software platform, with the developer joining the Dentally team. Gradually the Dentally team will transition the customer base fully on to the Dentally software.

To find out more about Dentally and to arrange a free demo, visit https://dentally.co/try-dentally or email hello@dentally.co.uk.

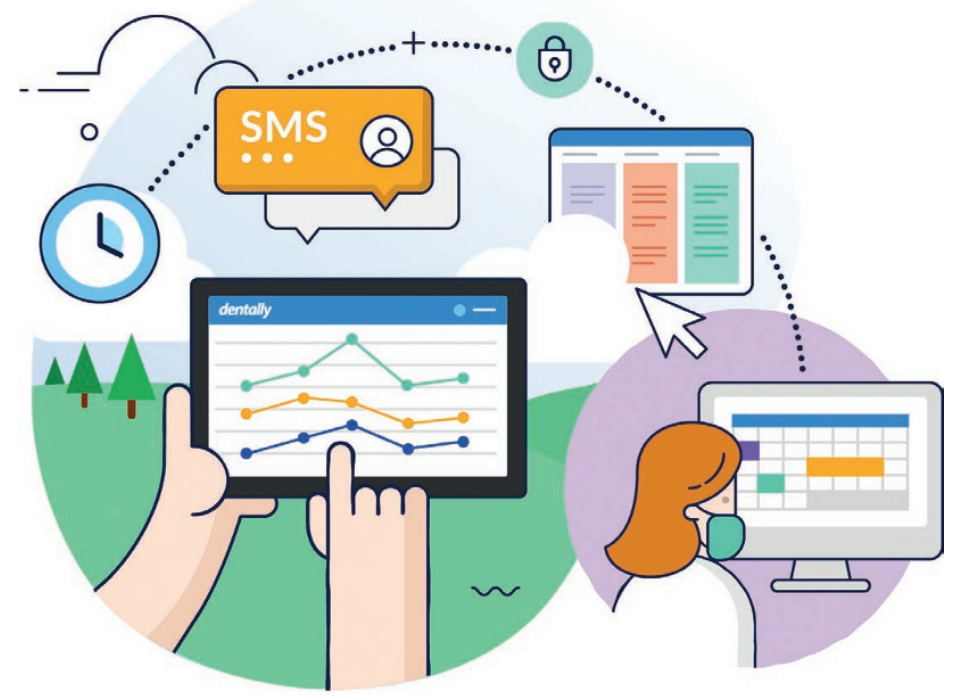

\section{Toothpaste with a 'heart of glass'}

A new toothpaste containing beneficial bio-glass called BioMin has become available. BioMin F allows a low dose of fluoride to be released steadily over 12 hours with calcium and phosphate creating tighter fluorapatite crystals that are far more resistant to acid attack than natural hydroxyapatite.

Chris Branfield, Principal Dentist at Castle Park Dental Care in Cottingham, said: 'A while ago I was asked to do a TV advert for Sensodyne. I liked their Repair and Protect toothpaste because it contains a bio-glass that sticks to surface like a protective layer and helps stop tooth sensitivity. However, there is now a new toothpaste containing an even more beneficial bio-glass called BioMin.

'Research shows that you benefit more from low doses of fluoride with a sustained release than from a high dose that washes away. It is not the amount of fluoride that counts but the length of time it is in contact with the teeth.

'That's where the BioMin F comes in! A low dose of fluoride released steadily over 12 hours with calcium and phosphate creates tighter fluorapatite crystals that are far more resistant to acid attack than natural hydroxyapatite.'

Branfield, also a founder member and trustee of the Dental Mavericks charity, adds: 'BioMin F sustains an optimum level of fluoride for up to 12 hours.'

More information on BioMin $\mathrm{F}$ is available at www.trycare. co.uk/biomin.

\section{Unrivalled alternative to amalgam}

New regulations mean that dental amalgam should not be used for dental treatment of pregnant or breastfeeding women, children under 15 years, or in deciduous teeth except when deemed strictly necessary by the dental practitioner based on the specific medical needs of the patient.

Therefore clinicians need to use an appropriate alternative to amalgam for Class I or II restorations in these situations.

GC's EQUIA Forte bulk fill glass hybrid restorative is the answer because of its enhanced physical properties, which deliver a stronger and superior posterior bulk fill restoration, and its 'GC pedigree', says the company. Unlike traditional GIC's, Equia Forte is recommended in its 'Instructions For Use' for Class I and II restorations (in both non-stress and certain stress bearing areas), intermediate restorations, Class V and root surface restorations, and core build-ups.

Compared with other restorative options Equia Forte gives a substantial time advantage, whilst its aesthetics widen the posterior restorative options enabling dentists to fulfil their patient's expectations. It also offers superior qualities of fluoride release and chemical bonding to all types of tooth structure creating a perfect seal against secondary caries, said the company. More information is available by contacting GC UK Ltd on 01908 218999, emailing info@gcukltd.co.uk or by visiting www.gceurope.com. 